\title{
Development of a thermostable microneedle patch for influenza vaccination
}

\author{
Matthew Mistilis, Andreas S Bommarius ${ }^{*}$, and Mark R. Prausnitz \\ School of Chemical and Biomolecular Engineering, Georgia Institute of Technology, 311 Ferst \\ Drive, Atlanta, GA 30332-0100 USA
}

\begin{abstract}
The goal of this study is to develop thermostable microneedle patch formulations for influenza vaccine that can be partially or completely removed from the cold chain. During vaccine drying associated with microneedle patch manufacturing, ammonium acetate and HEPES buffer salts stabilized influenza vaccine, surfactants had little effect during drying, drying temperature had weak effects on vaccine stability, and drying on polydimethylsiloxane led to increased stability compared to drying on stainless steel. A number of excipients, mostly polysaccharides and some amino acids, further stabilized the influenza vaccine during drying. Over longer time scales of storage, combinations of stabilizers preserved the most vaccine activity. Finally, dissolving microneedle patches formulated with arginine and calcium heptagluconate had no significant activity loss for all three strains of seasonal influenza vaccine during storage at room temperature for six months. We conclude that appropriately formulated microneedle patches can exhibit remarkable thermostability that could enable storage and distribution of influenza vaccine outside the cold chain.
\end{abstract}

\section{Background}

Influenza is a potentially life-threatening infectious disease that causes up to 5 million cases of severe illness worldwide during seasonal epidemics, while up to 500,000 people die from the disease each year. ${ }^{1}$ Annual vaccination against influenza is recommended by the Centers for Disease Control and Prevention (CDC; Atlanta, GA) for everyone 6 months of age and older. $^{2}$ The influenza virus is a spherical lipid membrane-enveloped virus. One of its 11 proteins ${ }^{3}$ is a spike protein, hemagglutinin (HA), which is the influenza vaccine's primary antigenic protein. ${ }^{2}$ This study was conducted using an inactivated purified subunit influenza vaccine where HA and neuraminidase (NA) are in rosette form.

Despite the wide availability of a relatively low-cost vaccine, seasonal influenza vaccination coverage for adults in the United States hovers around just 40\%. ${ }^{4}$ Two reasons for the low vaccination coverage are patients' needle phobia and inconvenience of travelling to a healthcare setting to receive the vaccine. ${ }^{5}$ Currently, the most common route of influenza vaccination is an intramuscular injection of an aqueous suspension of HA. This method has

\footnotetext{
*To whom correspondence should be addressed: andreas.bommarius@ chbe.gatech.edu (ASB), prausnitz@ gatech.edu (MRP). This potential conflict of interest has been disclosed and is overseen by Georgia Tech and Emory University.
} 
several drawbacks, such as a reliance on the cold-chain for transportation and storage, a possibility of needle-stick injuries, and patients' needle phobia. There are other routes of delivery such as nasal delivery and intradermal injection, which are used in recently approved products and also require cold-chain storage, administration by healthcare personnel and delivery methods that many people find unpleasant. ${ }^{2}$

We and others have proposed the use of microneedle patches for influenza vaccination ${ }^{6-10}$ because of the previously demonstrated benefits of less patient pain ${ }^{11}$, improved immune response $^{10}$, and possible self-administration ${ }^{12}$. In this way, microneedle patches may enable scenarios where vaccine can be picked up over-the-counter at stores or sent in the mail, so that people can vaccinate themselves and their families at home. An additional feature that would facilitate increased and simplified access to influenza vaccination using a microneedle patch would be reduced reliance on the cold-chain for transportation and storage, thus allowing vaccines to be stockpiled more easily and transported to vaccine recipients. Therefore, the goal of this study is to develop thermostable microneedle patch formulations that can be partially or completely removed from the cold chain.

Much knowledge about preserving vaccines in a dried state comes from the area of lyophilization. ${ }^{13}$ The vaccine antigen is typically formulated in the liquid state with various excipients such as buffer salts, bulking agents, and cryoprotectants, and then freeze-dried to form a solid powder. One common approach to vaccine stabilization is to incorporate the vaccine into an amorphous sugar glass. This approach has been taken by a number of investigators to stabilize influenza $\mathrm{HA}$ in the dry state by optimizing $\mathrm{pH}$, excipient composition, drying method, and other parameters. ${ }^{14}$ Previous work has shown that HA can be stabilized during air drying onto microneedles with sugars such as trehalose and methylcellulose ${ }^{15-16}$. However, we also showed that the inclusion of surfactant, while useful for coating metal microneedles, leads to matrix crystallization and HA activity loss. ${ }^{17}$ While sugars are commonly used stabilizing excipients other compounds, such as amino acids, have been examined as well. ${ }^{13}$

Guided by this previous work, in this study we determined the effect of formulation, drying, and storage conditions on the stability of a licensed sub-unit influenza vaccine during the manufacturing of microneedle patches. Initial screening was carried out by drying vaccine formulations suitable for microneedle patch fabrication and screening them for vaccine stability, which was followed by preparing actual microneedle patches using the best formulations and assessing stability during long-term storage.

\section{Materials and Methods}

\section{Vaccine and reagents}

Monovalent vaccine stocks were generously provided by Novartis Vaccines and Diagnostics (Cambridge, MA). All studies except for those involving trivalent patches were conducted using a monovalent stock of B/Brisbane/60/2008 influenza antigen. Trivalent patches included B/Brisbane/60/2008 as well as A/Brisbane/59/2007 (H1N1) and A/Victoria/ 210/2009 (H3N2) influenza antigens. All excipients were received from Sigma-Aldrich (St. 
Louis, MO), except for sodium heparin salt (Polysciences, Warrington, PA) and bovine serum albumin (EMD Millipore, Darmstadt, Germany).

\section{Formulations}

Vaccine stock solutions were received in phosphate-buffered saline (PBS). Monovalent stock of B/Brisbane $/ 60 / 2008$ received at a concentration of $360 \mu \mathrm{g} / \mathrm{mL}$ was buffer-exchanged using Vivaspin 500, $100 \mathrm{kDa}$ MWCO or Vivaspin 20, $10 \mathrm{kDa}$ MWCO centrifuge filters (Sartorius AG, Göttingen, Germany). Vaccine was concentrated at least 10-fold using the filters, then diluted with the desired buffer. This process was repeated 2-3 times. Finally, the same buffer was used to adjust the vaccine to the appropriate concentration for experiments, which was $60 \mu \mathrm{g} / \mathrm{mL}$, as determined by ELISA. All buffers were prepared at $300 \mathrm{mosmol} / \mathrm{L}$ and adjusted with $\mathrm{KOH}$ or $\mathrm{HCl}$ to $\mathrm{pH} 7.0$ - 7.4.

To formulate vaccine, dry excipients were weighed and placed into Type 1 glass vials with fused inserts (ChemGlass, Vineland, NJ) or Type 1 glass vials (Wheaton, Millville, NJ), to which buffer-exchanged vaccine stock solution was added. All formulations contained $1 \%$ w/v $250 \mathrm{kDa}$ sodium carboxymethyl cellulose ( $\mathrm{NaCMC}$ ). In the study on the effect of surfactant on vaccine stability, each surfactant was added so that the concentration was $50 \%$ higher than its critical micelle concentration (see Table S1 in Supporting Information). All formulations contained a total stabilizer excipient concentration of either $10 \%$ or $15 \% \mathrm{w} / \mathrm{v}$, where the concentration was split amongst up to four compounds, unless the stabilizer was not soluble to the desired level, in which case the concentration of the stabilizer was its solubility limit at $25^{\circ} \mathrm{C}$.

\section{Vaccine stability screening experiments}

To increase the throughput of formulation screening, drops of vaccine solution were dried on surfaces representative of coated metal microneedles or dissolving polymer microneedles. To simulate a metal microneedle drying surface, $6 \mathrm{~mm}$-diameter circular "chips" were cut from sheets of Type 301 stainless steel (McMaster, Atlanta, GA). To simulate the drying surface of dissolving microneedle molds, a thin film of Sylgard 184 (Dow Corning, Midland, MI) was cured, from which $6 \mathrm{~mm}$-diameter circular chips were removed with a hammer punch. Onto these surfaces were placed approximately $2 \mu \mathrm{L}$ droplets of formulated vaccine solution at a concentration of approximately $100 \mu \mathrm{g} / \mathrm{mL}$. Droplet weights were recorded on a Sartorius SE2 Ultra Micro Balance (Sartorius AG) for normalization to account for different actual masses administered in each droplet. The vaccine was allowed to dry at $15-25 \%$ relative humidity without added convection before chips were packaged in aluminum pouches (Oliver-Tolas Healthcare, Grand Rapids, MI) and sealed with an impulse heat-sealer (AIE-300, American International Electric, Industry, CA). All samples were stored with desiccant (Hammond Drierite, Xenia, $\mathrm{OH}$ ), unless otherwise noted. Chips were then stored for up to 182 days in Model 6020 environmental test chambers maintained at $5^{\circ} \mathrm{C}, 25^{\circ} \mathrm{C}$ or $40^{\circ} \mathrm{C}$ (Caron, Marietta, $\mathrm{OH}$ ). Vaccine activity remaining was calculated by comparing the dried sample to a positive control of similarly prepared vaccine that was not dried to account for any changes during formulation. 


\section{Microneedle patch preparation}

Dissolving microneedle patches were prepared using two separate solutions. The vaccine solution consisted of influenza vaccine, $1 \% \mathrm{w} / \mathrm{v} \mathrm{NaCMC}$, and $10 \% \mathrm{w} / \mathrm{v}$ of stabilizer in 150 $\mathrm{mM}$ ammonium acetate buffer. The backing solution consisted of polyvinyl alcohol, sucrose, and water in a mass ratio of $8: 6: 15$. The production procedure consisted of the following: 1 ) $25 \mu \mathrm{L}$ of vaccine solution was cast onto a polydimethylsiloxane (PDMS) microneedle mold, while vacuum was applied to help pull the vaccine solution into the microneedle cavities. 2) Excess vaccine solution was removed from the mold with a flat blade. 3) The vaccine solution was allowed to dry into the tips of the microneedle mold cavities. 4) Approximately $200 \mu \mathrm{L}$ of backing solution was cast onto the microneedle mold under vacuum, thus forming a complete microneedle patch (see Figure S1 in Supporting Information). Each patch was then stored in a desiccator at room temperature for 2 days before demolding with Scotch tape (3M, St. Paul, MN). Demolded patches were stored for up to 6 months at $25^{\circ} \mathrm{C}$ in an environmental stability chamber.

\section{ELISA assay}

Reconstituted vaccine samples were assayed for hemagglutinin activity using a sandwich enzyme-linked immunosorbent assay (ELISA). Polyclonal, strain-specific antibodies were received from the Center for Biologics Evaluation and Research (CBER) of the Food and Drug Administration (FDA; Silver Spring, MD). Antibodies were conjugated to horseradish peroxidase with a Lightning Link conjugation kit (Innova Biosciences, Cambridge, UK). Unformulated vaccine stock was serially diluted in phosphate-buffered saline with $0.5 \%$ Tween-20 (PBST) and used as a reference standard curve. After the prescribed storage time, chips and microneedle patches were placed into PBS so that the dried vaccine and excipients were redissolved. This solution was run parallel to the reference standard curve on an Immulon 2HB 96-well microplate (Thermo Scientific, Waltham, MA). The microplate was washed 3 times between each step with PBST with 3\% w/v bovine serum albumin. The HRP substrate reaction involved SureBlue Reserve TMB solution (KPL, Gaithersburg, MD), which was stopped with TMB Stop Solution (KPL). The microplate absorbance at $620 \mathrm{~nm}$ was read using an iMark plate reader (BioRad, Hercules, CA). The standard curve was fit to a four-parameter function using the Microplate Manager 6 software (BioRad). HA activity is presented as the percent of HA activity remaining for dried chip samples compared to liquid control solutions and as the total mass of active HA remaining in microneedle patches.

\section{Statistics}

All statistics were calculated using MiniTab software version 17 (MiniTab, State College, PA). All listed averages represent the arithmetic mean of the tested samples. Comparisons within individual samples were performed using an unpaired $t$-test with a significance cutoff of $p<0.05$. For comparisons between three or more samples, a one-way ANOVA was used or the data were fit to a general linear model, depending on the number of parameters varied, to determine significance. 


\section{Results}

\section{Drying time/temperature and buffer salts}

During microneedle patch manufacturing, a vaccine solution is typically dried on a microneedle surface or in a microneedle mold. To assess vaccine HA activity loss during this drying process, we dried solutions of $\mathrm{B} / \mathrm{Brisbane} / 60 / 2008$ influenza vaccine on stainless steel chips to simulate manufacturing of metal microneedles coated with vaccine and on PDMS chips to simulate manufacturing of dissolving polymer microneedles in molds. The vaccine was formulated with $\mathrm{NaCMC}$ (to increase viscosity), trehalose (to help stabilize the vaccine during drying, as shown in previous studies ${ }^{15}$ ) and one of four different buffer salts. Drying was performed at $4^{\circ} \mathrm{C}, 25^{\circ} \mathrm{C}$ and $40^{\circ} \mathrm{C}$ for 60,20 and $7 \mathrm{~min}$, respectively. Each chip was allowed to dry at the designated temperature until the sample was "dry," as determined by no further loss of mass due to water evaporation, i.e., equilibration with the surrounding air with humidity of $15-25 \%$ (data not shown). Upon drying, each sample was reconstituted and assayed for binding activity by ELISA.

As shown in Figure 1, HA activity loss varied approximately from $0 \%$ to $50 \%$, depending on vaccine formulation and drying conditions. Drying on stainless steel lead to more activity loss when compared to drying on PDMS (general linear model, $\mathrm{p}<0.001$ ). Choice of buffer salt also affected activity loss, where PBS performed the worst, and HEPES and ammonium acetate retained more vaccine activity compared to potassium diphosphate (general linear model, $\mathrm{p}<0.001$ and $\mathrm{p}<0.01$, respectively). Additional analysis showed that different drying temperatures (and thus different drying times, since drying was slower at lower temperature) had a significant effect on vaccine stability also (general linear model, $\mathrm{p}<$ 0.001), but no statistical difference was found when drying vaccine in ammonium acetate on PDMS at any temperature tested (ANOVA, $\mathrm{p}>0.85$ ). Based on these findings, we carried out the remaining experiments using vaccine solution buffer-exchanged into ammonium acetate buffer $(\mathrm{pH} 7.0)$ and dried on PDMS at room temperature.

\section{Surfactants}

Surfactants are often included in formulations used to coat microneedles. ${ }^{18}$ The effects of including surfactants in the vaccine solution formulation were therefore examined by choosing representative surfactants from several categories, including cationic, anionic, zwitterionic and non-ionic. The concentration of each surfactant was chosen to be 50\% higher than its critical micelle concentration (see Table S1 in Supporting Information). First, the effect of preparing liquid vaccine solutions containing surfactants was assessed, which showed that the addition of surfactant to the liquid formulations reduced HA activity in almost all cases by up to $\sim 50 \%$ (Figure 2). Then, the effect of drying these formulations was assessed. We found that there was no significant further loss of HA activity due to the drying in most cases (Figure 2).

Although surfactants did not generally affect HA stability during drying, the surfactants did generally reduce HA activity in the solution state before drying and previous studies have shown that surfactants can damage influenza vaccine during extended storage, possibly due to direct effects on the vaccine, such as solubilization of the viral membrane ${ }^{19}$, or indirect 
effects on the vaccine, such as increased crystallization of formulation components ${ }^{17}$. For this reason, further experiments were performed without surfactant in the formulation.

\section{Stabilizer screening}

Our next step was to screen excipients that further stabilize HA activity during drying. Formulated vaccine was dried on PDMS chips and stored for $24 \mathrm{~h}$ at room temperature with desiccant. As shown in Figure 3, there was a broad range of effects, spanning from almost complete loss of activity to almost complete retention of activity. These HA activity levels were compared to that of vaccine that was dried in the absence of excipients (i.e., the bar on the far right of the graph) and classified into three categories: compounds that stabilized the vaccine (i.e., greater stability than the unformulated control, Student's t-test, $\mathrm{p}<0.05$ ), compounds that had no significant effect ( $p>0.05$ ), and compounds that destabilized the vaccine ( $\mathrm{p}<0.05)$. Most of the compounds that stabilized HA activity were carbohydrates along with a few amino acids.

To identify formulations that stabilize HA not only during drying, but also during subsequent storage, dried vaccine samples were stored for 1 week at $40^{\circ} \mathrm{C}$ as a second level of screening. Because there can be improved effects when multiple stabilizers are used together ${ }^{20}$, we selected the best stabilizers from the initial screen and assessed their effect alone or in pairwise combination with each other. This second, more-stringent screen identified individual stabilizers that continued to be effective and identified stabilizer combinations that were effective as well (Figure 4). In most cases, stabilizer combinations were no better than at least one of the individual stabilizers, except for two combinations (Figure 4).

Based on the one-week screening study, vaccine formulations were prepared using the top five stabilizers, as well as each two-stabilizer combination of these excipients, dried and stored at $40^{\circ} \mathrm{C}$ for up to one month. While most formulations were stable up to one week, most of them also lost substantial HA activity at one month (Figure 5). Three combinations, however, had no significant activity loss during the one month of storage. These combinations were trehalose/sucrose, sucrose/arginine, and arginine/heptagluconate.

\section{Long-term stability of chips}

Using the optimal vaccine formulations identified in the one-month screen, we carried out a six-month screen using those excipients in pairwise combination at formulation ratios of 90:10, 50:50, and 10:90, as well as combinations of three excipients and all four excipients combined in equal amounts (Figure 6). All the formulations tested in this study were generally stable for three months, but at six months HA activity was lost in many formulations. Among the two-stabilizer combinations of formulations, there was a significant effect of formulation ratio when varied between 90:10, 50:50 and 10:90 (ANOVA, $\mathrm{p}<0.05$ ) except for arginine and calcium heptagluconate at a ratio of either 50:50 or 10:90 ( $p>0.092$ ), where 50:50 ratio mixtures performed better than the other two ratios tested. At the six-month time point, only formulations containing arginine and calcium heptagluconate among the two-, three- or four-fold excipient combinations, as well as the 
trehalose/sucrose/arginine formulation, had no significant loss in HA activity relative to day 0 (Student's t-test, $\mathrm{p}<0.05$ ).

\section{Packaging humidity}

To examine the effect of humidity during storage, several single-excipient formulations were dried on PDMS chips and stored at $40^{\circ} \mathrm{C}$ for up to 16 weeks in aluminum pouches with either desiccant, to maintain a humidity approaching zero, or conditioned silica gel pouches, to maintain a relative humidity of $70 \%$. As seen in Figure 7, desiccated samples were generally stable, whereas samples stored at elevated humidity rapidly lost HA activity.

\section{Long-term stability of microneedle patches}

Guided by screening studies carried out on chips, we prepared microneedle patches containing monovalent or trivalent influenza vaccine using various formulations that were stored with desiccant at $25^{\circ} \mathrm{C}$ for up to six months. Monovalent patches, incorporating HA from $\mathrm{B} / \mathrm{Brisbane} / 60 / 2008$, were formulated with the top three two-stabilizer combinations as well as the component individual stabilizers. As shown in Figure 8, microneedle patches without stabilizing excipients lost activity during the initial production phase (i.e. at day 0 ) and further lost activity during subsequent storage for six months (Student's t-test, $\mathrm{p}<$ 0.001). The formulations containing individual and paired combinations of stabilizing excipients performed better both during initial production and subsequent storage (Figure 8). The only stabilizer combination that had no significant loss of HA activity was arginine and calcium heptagluconate.

Finally, trivalent influenza vaccine patches were produced with a target of a full human dose, i.e., $15 \mu \mathrm{g}$ of HA per strain per patch of A/Brisbane/59/2007 (H1N1), A/Victoria/210/2009 (H3N2) and B/Brisbane/60/2008. As shown in Figure 9, these patches were at or slightly below the target dose upon fabrication. After three months of storage at $25^{\circ} \mathrm{C}$, all patch formulations had no significant loss of HA activity (ANOVA, $p>0.085$ ). The stability of influenza antigen varied by strain over 6 months of storage, but the arginine/heptagluconate formulation had no significant loss of HA activity for any of the three strains. This suggests that this microneedle patch formulation could enable long-term storage of trivalent influenza vaccine without refrigeration.

\section{Discussion}

This study seeks to develop a thermostable microneedle patch for influenza vaccination. Through a series of screening and optimization studies to develop formulation compositions and manufacturing parameters that enhance thermostability of the HA antigen in influenza vaccine, we developed microneedle patch formulations that protected the influenza vaccine antigen during six months of storage at room temperature.

In the study design, we expected two timescales of HA activity loss. The first is the initial drying phase where the liquid formulation is allowed to air-dry over the course of minutes to hours, thereby stressing the antigen as water is removed. The second timescale occurs over days to months, as the dried antigen and its matrix can slowly change during storage. The study plan therefore included initial screening of formulations and manufacturing conditions 
that protected the vaccine during the initial drying phase, and then built on those results to further develop formulations that protected the vaccine during storage.

Much of the optimization was carried out on "chips" that simulated the manufacturing process without the need to go through the more laborious process of making actual microneedle patches. Advantages of this approach included ease of operation, control of antigen loading, and reduced amount of antigen required per test. The PDMS chips were representative of PDMS molds used during manufacturing of dissolving microneedles ${ }^{21}$ and the stainless steel chips were representative of the metal microneedles used during manufacturing of coated microneedles. Based on the first set of experiments, we identified drying on PDMS at room temperature using ammonium acetate buffer as our baseline operating condition. We chose PDMS as the drying substrate because HA activity was more stable on PDMS than on stainless steel, and because we are primarily interested in making dissolving microneedle patches that generate no sharps waste, which are manufactured using PDMS molds. We chose room temperature, because there was not a significant effect of temperature on HA activity when ammonium acetate was the buffer, and room temperature is simpler to implement than cooled or heated drying, and is faster than drying at low temperature. Although HEPES buffer performed similarly well, we chose ammonium acetate buffer because it maintained HA activity during drying and, as a volatile buffer, it will partially evaporate during drying, thereby leaving fewer crystals. ${ }^{23} \mathrm{PBS}$ performed the worst, perhaps because of $\mathrm{pH}$ drops when dibasic phosphate precipitates before monobasic phosphate. $^{24}$

Surfactants were found to destabilize HA activity, especially in liquid formulation before drying. Previous work has further shown that surfactants can cause crystallization in the microneedle coating matrix during storage and cause damage to HA activity. ${ }^{17}$ We therefore chose not to include surfactants in our formulation, since they are not typically needed when molding dissolving microneedles, although they are often used when coating metal microneedles. ${ }^{25}$

Using these baseline operating conditions, we screened a large number of excipients selected to stabilize HA activity during drying and subsequent storage. Candidate excipients were selected from the literature and past data from our laboratory showing stabilization of HA and other proteins and vaccines, included conventionally used stabilizers as well as other compounds not well known for their stabilizing properties. We found that approximately $45 \%$ of the excipients tested provided significant stabilizing effects, almost $20 \%$ destabilized HA and the remainder had no significant effect when compared to drying the vaccine with no additional excipients. The large majority of stabilizers were carbohydrates, including small saccharides, polyols, and long-chain polysaccharides. Two notable exceptions were the stabilizing amino acids, arginine and histidine.

We next tested combinations of the best-performing stabilizers, some of which performed better than their individual excipient components. The best-performing formulations generally contained arginine and calcium heptagluconate either in pairwise combination or as components of formulations containing additional excipients as well, such as both trehalose and sucrose. Considering the detailed composition of the formulation, we generally 
found among a set of two excipient combinations, a composition of 50:50 retained the most antigen activity.

Finally, we prepared dissolving microneedle patches based on these screening studies on PDMS chips. Trivalent influenza vaccine patches formulated with arginine and calcium heptagluconate had no significant loss of HA activity of any of the three vaccine strains after storage for six months at $25^{\circ} \mathrm{C}$. This suggests that a thermostable microneedle patch for influenza vaccination could be developed without the need for cold-chain storage and distribution.

The development of a thermostable influenza vaccine patch could facilitate greater influenza vaccination coverage during both seasonal and pandemic influenza scenarios. The cold chain contributes substantially to the cost of storing and transporting vaccine doses. ${ }^{26-27} \mathrm{In}$ addition, thermostability is important to our long-term vision of a self-administered microneedle patch for influenza vaccination, ${ }^{12}$ where vaccine could be stored on nonrefrigerated shelves in the pharmacy and could be mailed to patients at home without ice packs.

\section{Conclusions}

This study has the objective to develop a thermostable microneedle patch for influenza vaccination. Initial studies focusing on maintaining stability during the drying of influenza vaccine associated with microneedle patch manufacturing determined that HA activity was higher after drying on PDMS than on stainless steel, was only sometimes significantly affected by drying temperature at $4^{\circ} \mathrm{C}, 25^{\circ} \mathrm{C}$ or $40^{\circ} \mathrm{C}$, was more effectively stabilized by ammonium acetate and HEPES buffers than phosphate-based buffers, and was destabilized by addition of surfactants. Subsequent studies assessed the ability of 60 excipients to stabilize HA activity during drying and subsequent storage at elevated temperature. The best excipients, and excipient combinations, were then identified as those that maintained HA activity for up to six months at $40^{\circ} \mathrm{C}$ when stored with desiccant. The best performing stabilizer combination was arginine/heptagluconate in a 50:50 or 10:90 ratios. As a final test of stability, microneedle patches were stored at room temperature for up to six months, during which essentially all HA activity was retained with the arginine/heptagluconate formulation. Additional experiments are needed to determine the mechanistic basis for these stabilizing effects. We conclude that the formulations developed here may enable development of a thermostable microneedle patch for influenza vaccination to improve future coverage during seasonal and pandemic vaccination.

\section{Supplementary Material}

Refer to Web version on PubMed Central for supplementary material.

\section{Acknowledgments}

We thank Novartis for generously providing monovalent influenza vaccine stock. This work was supported in part by the National Institutes of Health. We thank Polo Gaputan and Miraj Desai for their work on this project. The work was carried out in the Center for Drug Design, Development and Delivery, and the Institute for Bioengineering and Bioscience at the Georgia Institute of Technology. Matthew Mistilis, Andreas Bommarius and 
Mark Prausnitz are inventors of patent(s) that have been or may be licensed to companies developing microneedlebased products, and Mark Prausnitz is a paid advisor to companies developing microneedle-based products and is a founder/shareholder of companies developing microneedle-based products.

\section{References}

1. World Health Organization Influenza Fact Sheet. http://www.who.int/mediacentre/factsheets/ fs211/en/index.html (accessed 07/1/14)

2. Grohskopf L, et al. Prevention and control of seasonal influenza with vaccines: Recommendations of the Advisory Committee on Immunization Practices (ACIP) — United States, 2014-15 Influenza Season. Morbidity and Mortality Weekly Report. 2014; 63:691-697. [PubMed: 25121712]

3. Ghedin E, Sengamalay NA, Shumway M, Zaborsky J, Feldblyum T, Subbu V, Spiro DJ, Sitz J, Koo H, Bolotov P, Dernovoy D, Tatusova T, Bao Y, St George K, Taylor J, Lipman DJ, Fraser CM, Taubenberger JK, Salzberg SL. Large-scale sequencing of human influenza reveals the dynamic nature of viral genome evolution. Nature. 2005; 437:1162-1166. [PubMed: 16208317]

4. Santibanez, TA., Zhai, Y., Singleton, JA., et al. Flu Vaccination Coverage, United States, 2012-13 Influenza Season. http://www.cdc.gov/flu/fluvaxview/coverage-1213estimates.htm (accessed 7/1/14)

5. Taddio A, Ipp M, Thivakaran S, Jamal A, Parikh C, Smart S, Sovran J, Stephens D, Katz J. Survey of the prevalence of immunization non-compliance due to needle fears in children and adults. Vaccine. 2012; 30:4807-12. [PubMed: 22617633]

6. Sullivan SP, Koutsonanos DG, Martin MD, Lee JW, Zarnitsyn V, Choi SO, Murthy N, Compans RW, Skountzou I, Prausnitz MR. Dissolving polymer microneedle patches for influenza vaccination. Nature Medicine. 2010; 16:915-U116.

7. Kommareddy S, Baudner BC, Oh S, Kwon S-Y, Singh M, O'Hagan DT. Dissolvable microneedle patches for the delivery of cell-culture-derived influenza vaccine antigens. Journal of Pharmaceutical Sciences. 2012; 101:1021-1027. [PubMed: 22190403]

8. Kang SM, Song JM, Kim YC. Microneedle and mucosal delivery of influenza vaccines. Expert Review of Vaccines. 2012; 11:547-60. [PubMed: 22697052]

9. Matsuo K, Hirobe S, Yokota Y, Ayabe Y, Seto M, Quan Y-S, Kamiyama F, Tougan T, Horii T, Mukai Y, Okada N, Nakagawa S. Transcutaneous immunization using a dissolving microneedle array protects against tetanus, diphtheria, malaria, and influenza. Journal of Controlled Release. 2012; 160:495-501. [PubMed: 22516091]

10. Koutsonanos, D., Compans, R., Skountzou, I. Targeting the skin for microneedle delivery of influenza vaccine. In: Katsikis, PD.Schoenberger, SP., Pulendran, B., editors. Crossroads Between Innate and Adaptive Immunity IV. Vol. 785. Springer; New York: 2013. p. 121-132.

11. Gill HS, Denson DD, Burris BA, Prausnitz MR. Effect of microneedle design on pain in human volunteers. Clinical Journal of Pain. 2008; 24:585-594. [PubMed: 18716497]

12. Norman JJ, Arya JM, McClain MA, Frew PM, Meltzer MI, Prausnitz MR. Microneedle patches: Usability and acceptability for self-vaccination against influenza. Vaccine. 2014; 32:1856-1862. [PubMed: 24530146]

13. Pikal, MJ. Mechanisms of protein stabilization during freeze-drying storage: The relative protection by stabilizing additives. In: Rey, L., May, JC., editors. Freeze-drying/lyophilization of pharmaceutical and biological products. 3rd. CRC Press; 2010.

14. Amorij JP, Huckriede A, Wischut J, Frijlink HW, Hinrichs WLJ. Development of stable influenza vaccine powder formulations: Challenges and possibilities. Pharmaceutical Research. 2008; 25:1256-1273. [PubMed: 18338241]

15. Kim YC, Quan FS, Compans RW, Kang SM, Prausnitz MR. Formulation and coating of microneedles with inactivated influenza virus to improve vaccine stability and immunogenicity. Journal of Controlled Release. 2010; 142:187-195. [PubMed: 19840825]

16. Chen X, Fernando GJP, Crichton ML, Flaim C, Yukiko SR, Fairmaid EJ, Corbett HJ, Primiero CA, Ansaldo AB, Frazer IH, Brown LE, Kendall MAF. Improving the reach of vaccines to lowresource regions, with a needle-free vaccine delivery device and long-term thermostabilization. Journal of Controlled Release. 2011; 152:349-355. [PubMed: 21371510] 
17. Choi HJ, Bondy BJ, Yoo DG, Compans RW, Kang SM, Prausnitz MR. Stability of whole inactivated influenza virus vaccine during coating onto metal microneedles. Journal of Controlled Release. 2013; 166:159-71. [PubMed: 23246470]

18. Kim YC, Quan FS, Compans RW, Kang SM, Prausnitz MR. Formulation of microneedles coated with influenza virus-like particle vaccine. AAPS PharmSciTech. 2010; 11:1193-1201. [PubMed: 20676947]

19. Nazari M, Kurdi M, Heerklotz H. Classifying surfactants with respect to their effect on lipid membrane order. Biophysical Journal. 2012; 102:498-506. [PubMed: 22325272]

20. Schlehuber LD, McFadyen IJ, Shu Y, Carignan J, Duprex WP, Forsyth WR, Ho JH, Kitsos CM, Lee GY, Levinson DA, Lucier SC, Moore CB, Nguyen NT, Ramos J, Weinstock BA, Zhang J, Monagle JA, Gardner CR, Alvarez JC. Towards ambient temperature-stable vaccines: The identification of thermally stabilizing liquid formulations for measles virus using an innovative high-throughput infectivity assay. Vaccine. 2011; 29:5031-5039. [PubMed: 21616113]

21. Lee JW, Park JH, Prausnitz MR. Dissolving microneedles for transdermal drug delivery. Biomaterials. 2008; 29:2113-2124. [PubMed: 18261792]

22. Gill HS, Prausnitz MR. Coated microneedles for transdermal delivery. Journal of Controlled Release. 2007; 117:227-237. [PubMed: 17169459]

23. Williams R, Lyman C. A neutral buffered standard for hydrogen ion work and accurate titrations which can be prepared in one minute. Journal of the American Chemical Society. 1932; 54:19111912.

24. Williams-Smith DL, Bray RC, Barber MJ, Tsopanakis AD, Vincent SP. Changes in apparent pH on freezing aqueous buffer solutions and their relevance to biochemical electron-paramagneticresonance spectroscopy. Biochem J. 1977; 167:593-600. [PubMed: 23760]

25. Gill HS, Prausnitz MR. Coating formulations for microneedles. Pharmaceutical Research. 2007; 24:1369-1380. [PubMed: 17385011]

26. Wolfson LJ, Gasse F, Lee-Martin S, et al. Estimating the costs of achieving the WHO-UNICEF Global Immunization Vision and Strategy, 2006-2015. Bulletin of The World Health Organization. 2008; 86:27-39. [PubMed: 18235887]

27. Brandau DT, Jones LS, Wiethoff CM, Rexroad J, Middaugh CR. Thermal stability of vaccines. Journal of Pharmaceutical Sciences. 2003; 92:218-231. [PubMed: 12532371] 

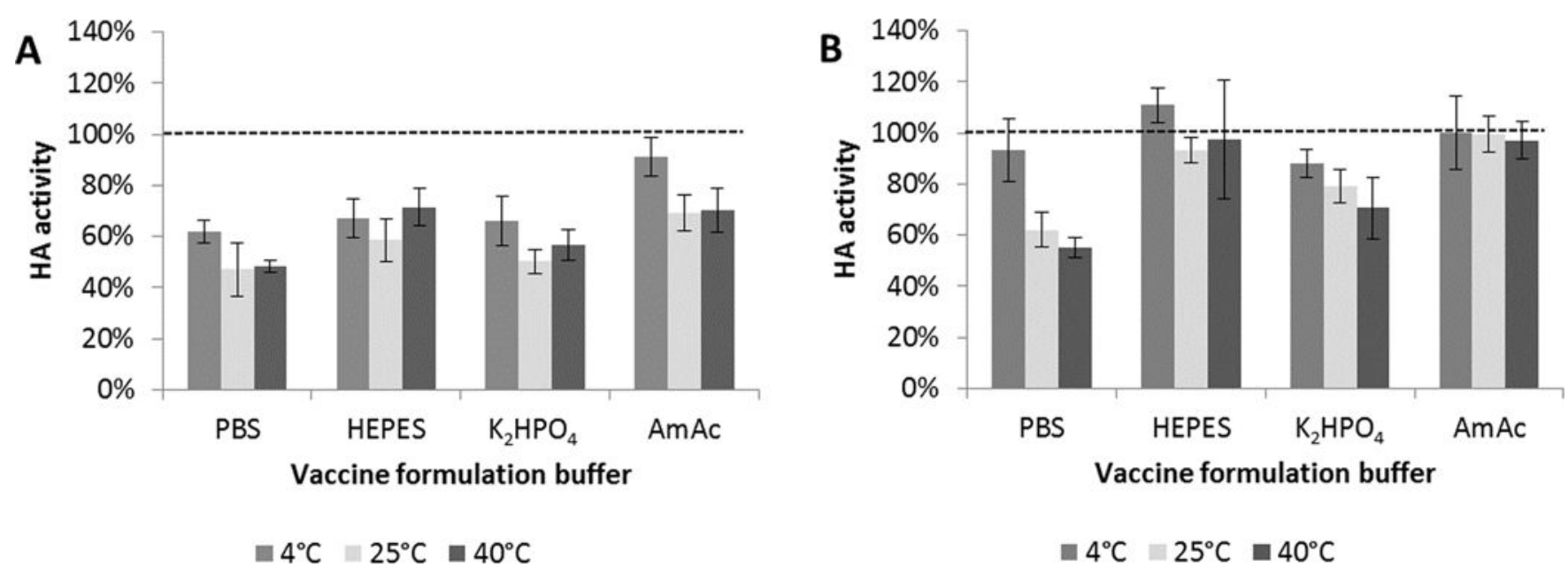

FIGURE 1.

Effect of formulation buffer, drying temperature and drying substrate on HA activity after drying influenza trehalose was dried on (A) stainless steel (SS) or (B) polydimethylsiloxane (PDMS) chips at $4^{\circ} \mathrm{C}, 25^{\circ} \mathrm{C}$ or $40^{\circ} \mathrm{C}$ and assayed for HA activity after $24 \mathrm{~h}$. PBS = phosphate-buffered saline; HEPES = 4-(2-hydroxyethyl)-1-piperazineethanesulfonic acid; $\mathrm{K}_{2} \mathrm{HPO}_{4}=$ potassium phosphate dibasic; AmAc $=$ ammonium acetate. $\mathrm{HA}$ activity is shown as a percentage of concentrated stock vaccine solution after buffer exchanging to the respective vaccine on chips until "dry". Vaccine (B/Brisbane/60/2008) formulated with $15 \%$ w/v buffer. Data represent averages of $n=6$ replicates, with standard deviation bars shown. 


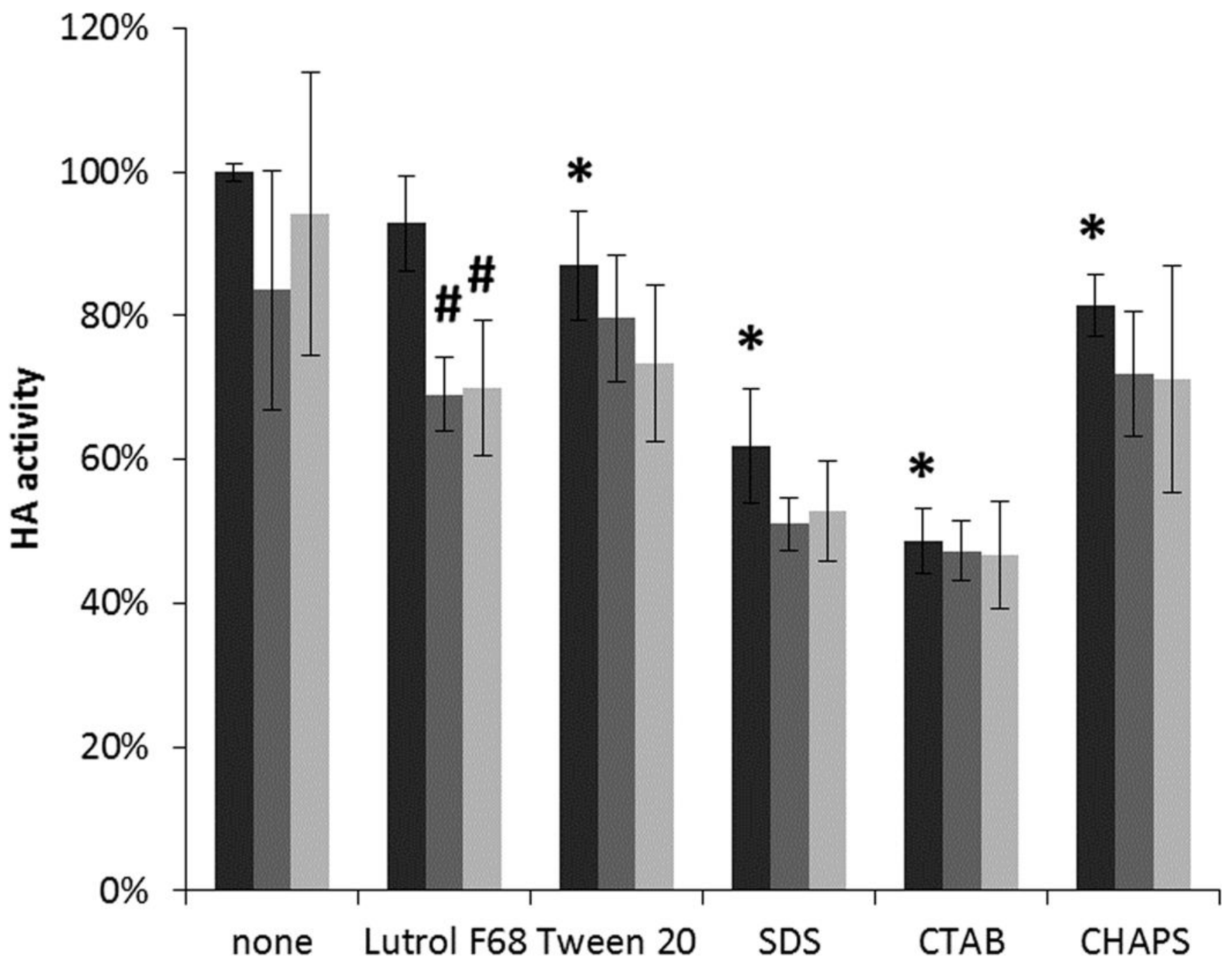

\section{Vaccine formulation surfactant}

\section{- Liquid $\quad$ SS $\quad$ PDMS}

FIGURE 2.

Effect of surfactant on HA activity after drying influenza vaccine on PDMS or stainless steel chips at $25^{\circ} \mathrm{C}$ until "dry". Vaccine (B/Brisbane/60/2008) was formulated with $15 \%$ w/v trehalose in ammonium acetate buffer. Asterisk (*) represents significant loss of HA activity in the liquid formulation containing surfactant compared to vaccine stock solution (Student's t-test, $\mathrm{p}<0.05$ ). Hash (\#) represents significant loss of HA activity in the dried formulation compared to the liquid formulation containing surfactant (Student's t-test, $\mathrm{p}<0.05$ ). HA activity is shown as a percentage of activity of concentrated stock vaccine solution with the corresponding surfactant. Data represent averages of $n=6$ replicates, with standard 
deviation bars shown. Surfactants and their critical micelle concentrations can be found in Table S1 in Supporting Information. 


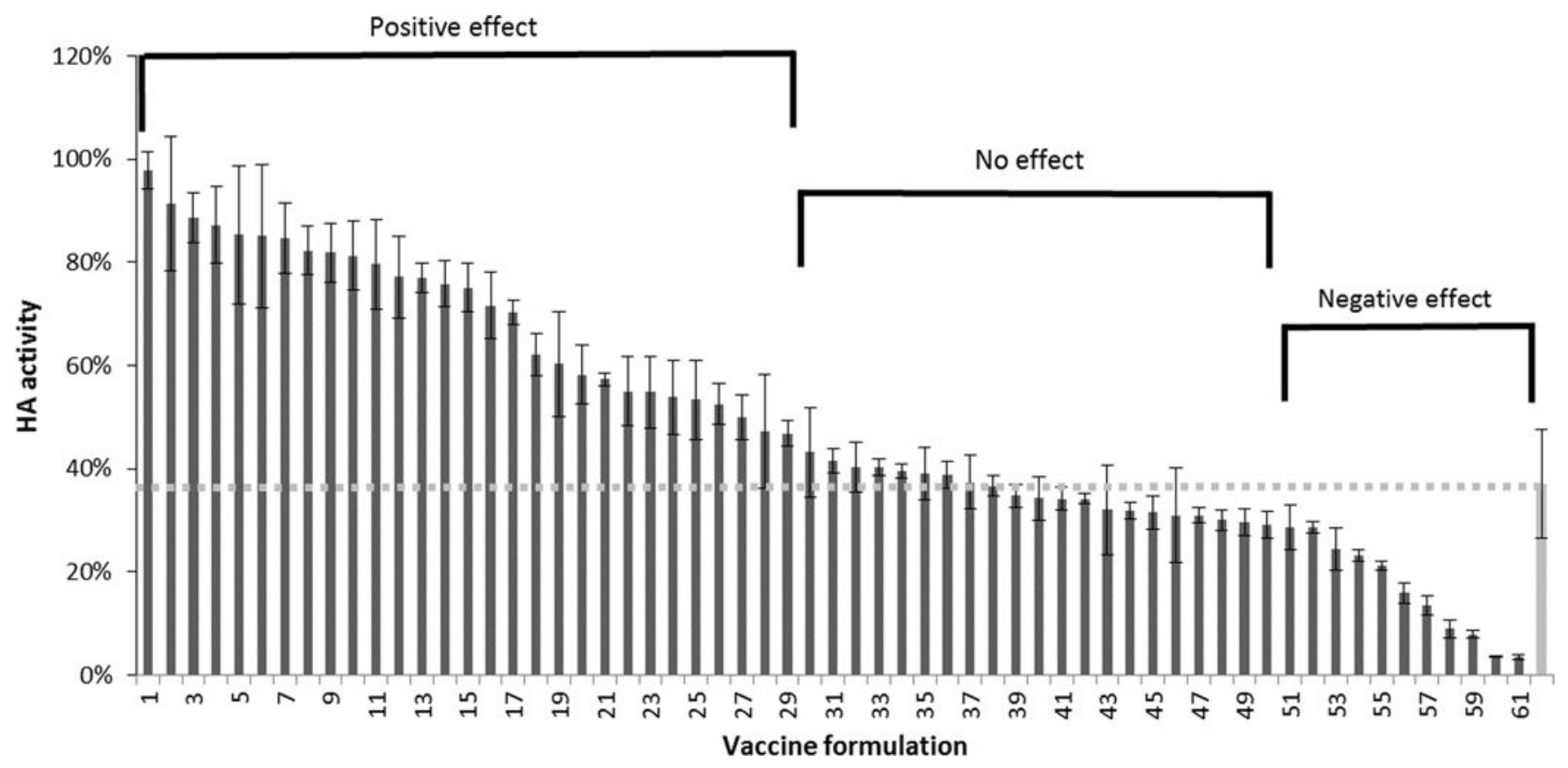

FIGURE 3.

Effect of stabilizing excipients on HA activity after drying influenza vaccine on PDMS chips and storing with desiccant for $24 \mathrm{~h}$ at room temperature $\left(25^{\circ} \mathrm{C}\right)$. Vaccine $(\mathrm{B} / \mathrm{Brisbane} /$ $60 / 2008$ ) was formulated with individual excipients at a concentration of $15 \% \mathrm{w} / \mathrm{v}$ in ammonium acetate buffer (See Table S2 in Supporting Information for key to excipient list). Excipients had a positive effect (i.e., HA activity was significantly greater than the dried, unformulated vaccine indicated by the gray bar on the far right; Student's t-test $p<0.05$ ), no significant effect $(p>0.05)$ or a negative effect $(p<0.05)$. HA activity is shown as a percentage of concentrated stock vaccine solution. Data represent averages of $n=6$ replicates, with standard deviation bars shown (Student's t-test, $\mathrm{p}<0.05$ ). 


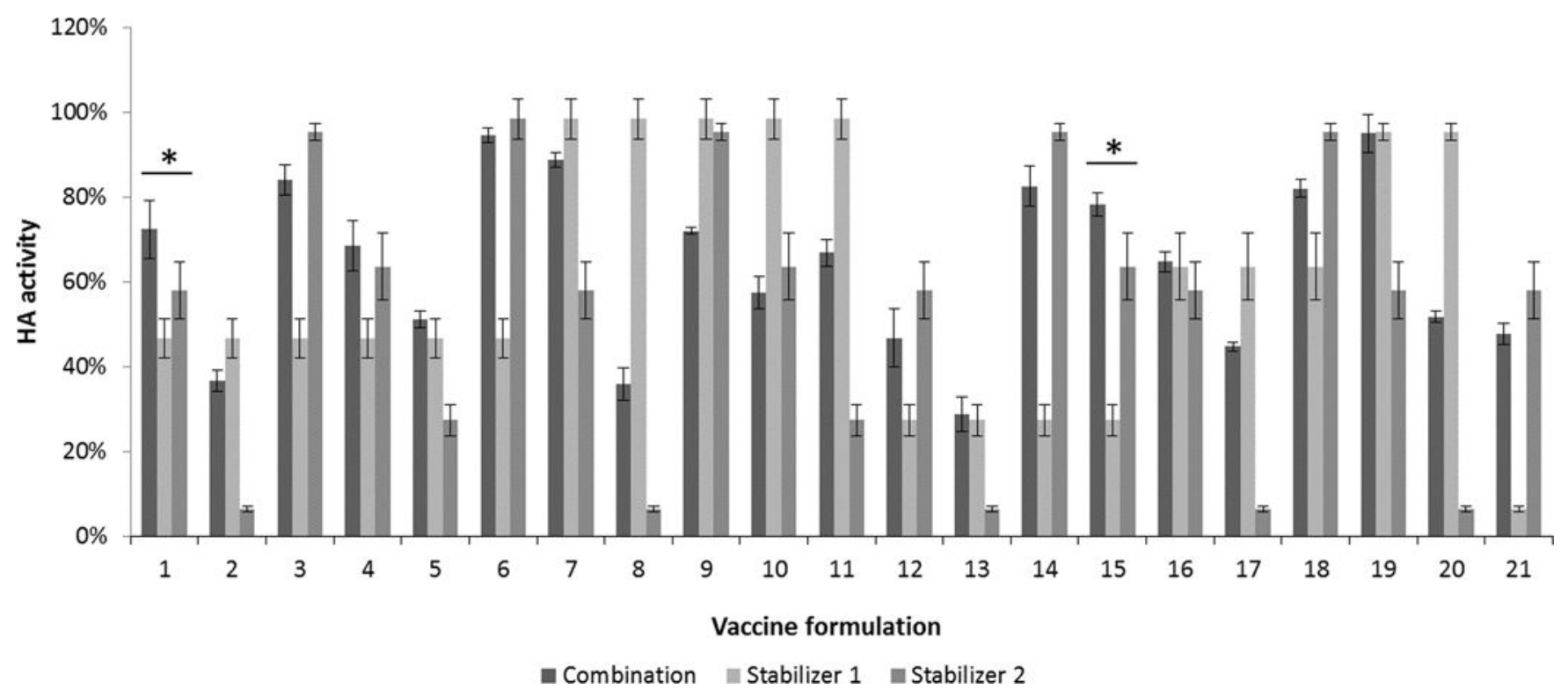

FIGURE 4.

Effect of combinations of stabilizing excipients on HA activity after drying influenza vaccine on PDMS chips and storing with desiccant for one week at $40^{\circ} \mathrm{C}$. Vaccine (B/ Brisbane/60/2008) was formulated with either one or two stabilizing excipients at a total stabilizer concentration of $15 \% \mathrm{w} / \mathrm{v}$ in ammonium acetate buffer (See Table S3 in Supporting Information for key to excipient list). In each set of bars, the first bar shows HA activity for the combination formulation, the second bar shows HA activity for formulation with just the first stabilizing excipient and the third bar shows HA activity for formulation with just the second stabilizing excipient. Asterisk (*) indicates combination-excipient formulations with significantly higher HA activity compared to either associated individualexcipient formulations (Student's t-test, $\mathrm{p}<0.05$ ). HA activity is shown as a percentage of concentrated stock vaccine solution. Data represent averages of $n=5$ replicates, with standard deviation bars shown. 


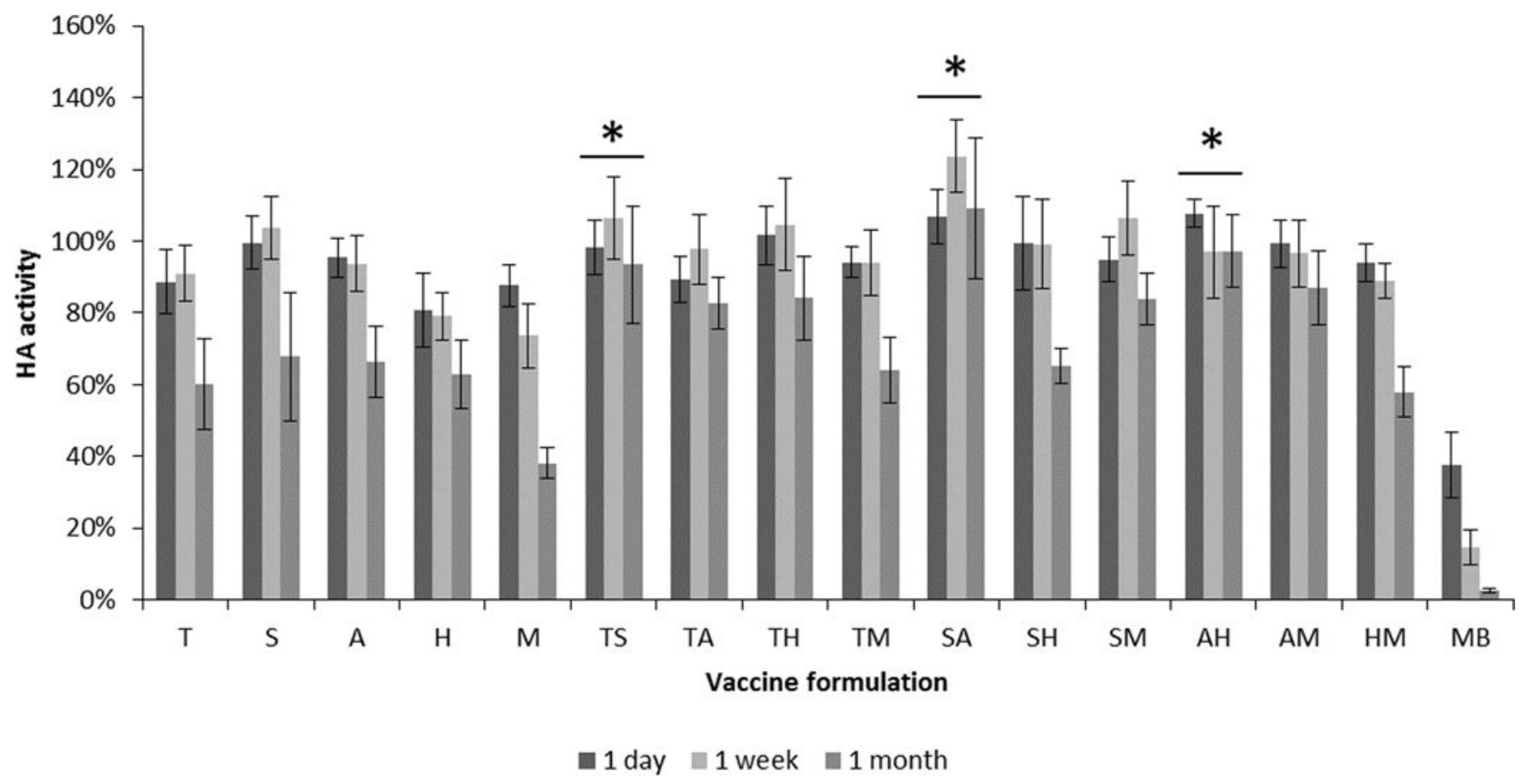

FIGURE 5.

Effect of stabilizing excipients on HA activity after drying influenza vaccine on PDMS chips and storing with desiccant for up to one month at $40^{\circ} \mathrm{C}$. Vaccine (B/Brisbane/60/2008) was formulated with individual or pair-wise combinations of stabilizing excipients at a total stabilizer concentration of $15 \% \mathrm{w} / \mathrm{v}$ in ammonium acetate buffer. HA activity is shown as a percentage of concentrated stock vaccine solution. $\mathrm{T}=$ trehalose, $\mathrm{S}=$ sucrose, $\mathrm{A}=$ arginine, $\mathrm{H}=$ calcium heptagluconate, $\mathrm{M}=$ maltodextrin $13, \mathrm{MB}=$ monobulk. Asterisk (*) indicates no statistically different HA activity amongst the three time points (Student's t-test, $\mathrm{p}<$ 0.05). Bars represent averages of $n=5$ replicates; with standard deviation bars shown. 


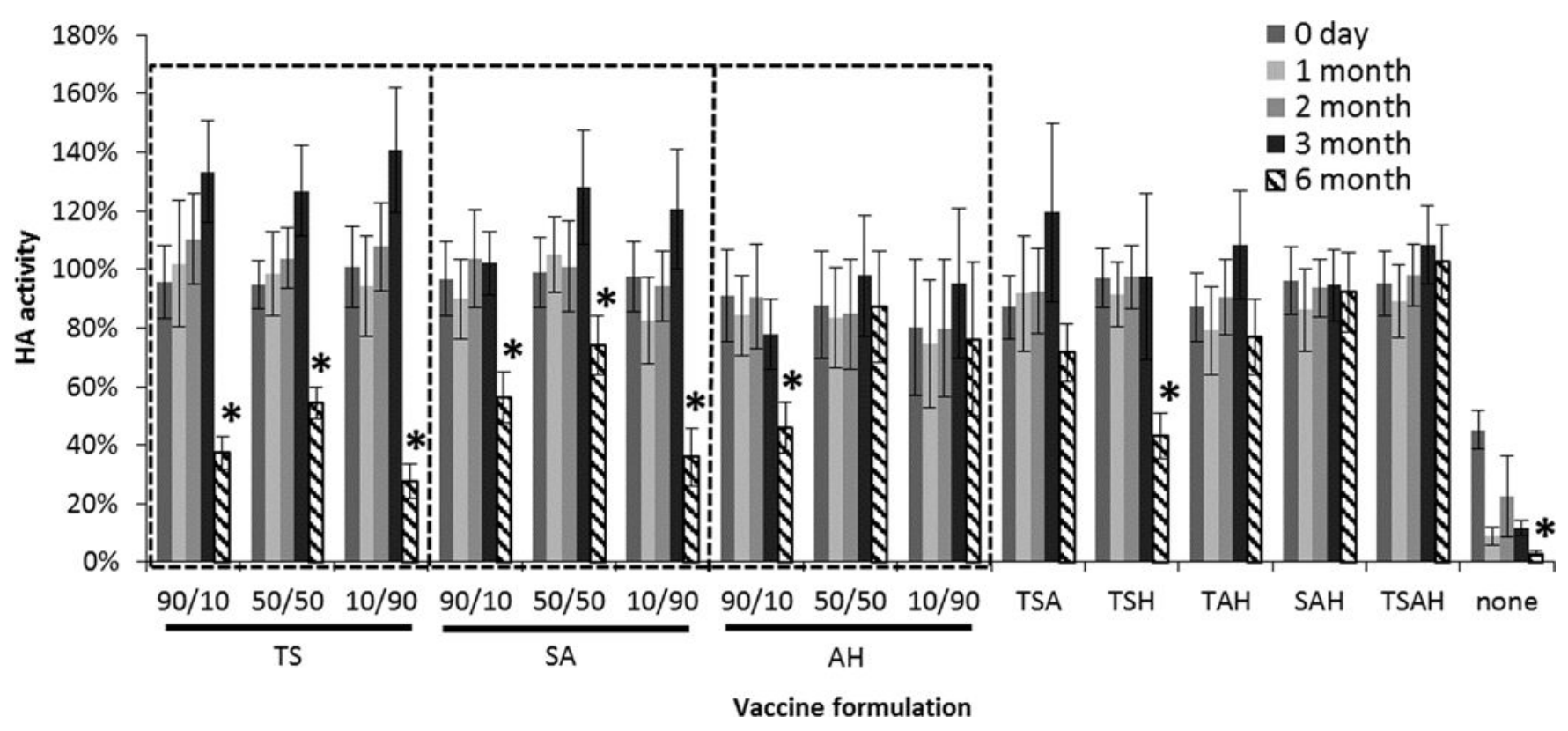

FIGURE 6.

Effect of stabilizing excipient combinations on HA activity after drying influenza vaccine on PDMS chips and storing with desiccant for up to six months at $40^{\circ} \mathrm{C}$. Vaccine (B/Brisbane/ 60/2008) was formulated with two, three or four stabilizing excipients at a total stabilizer concentration of $10 \% \mathrm{w} / \mathrm{v}$ in ammonium acetate buffer. For the two-stabilizer combinations, the concentration ratio is shown, where the first number corresponds to the first excipient listed below it on the $\mathrm{x}$ axis. The combinations of three or four stabilizers all contained equal amounts of each excipient. $\mathrm{T}=$ trehalose, $\mathrm{S}=$ sucrose, $\mathrm{A}=$ arginine, $\mathrm{H}=$ sodium heptagluconate. Asterisk $(*)$ indicates HA activity significantly decreased from HA activity on day 0 (Student's t-test, $\mathrm{p}<0.05$ ). HA activity is shown as a percentage of concentrated stock vaccine solution. Data represent averages of $\mathrm{n}=12$ replicates, with standard deviation bars shown. 

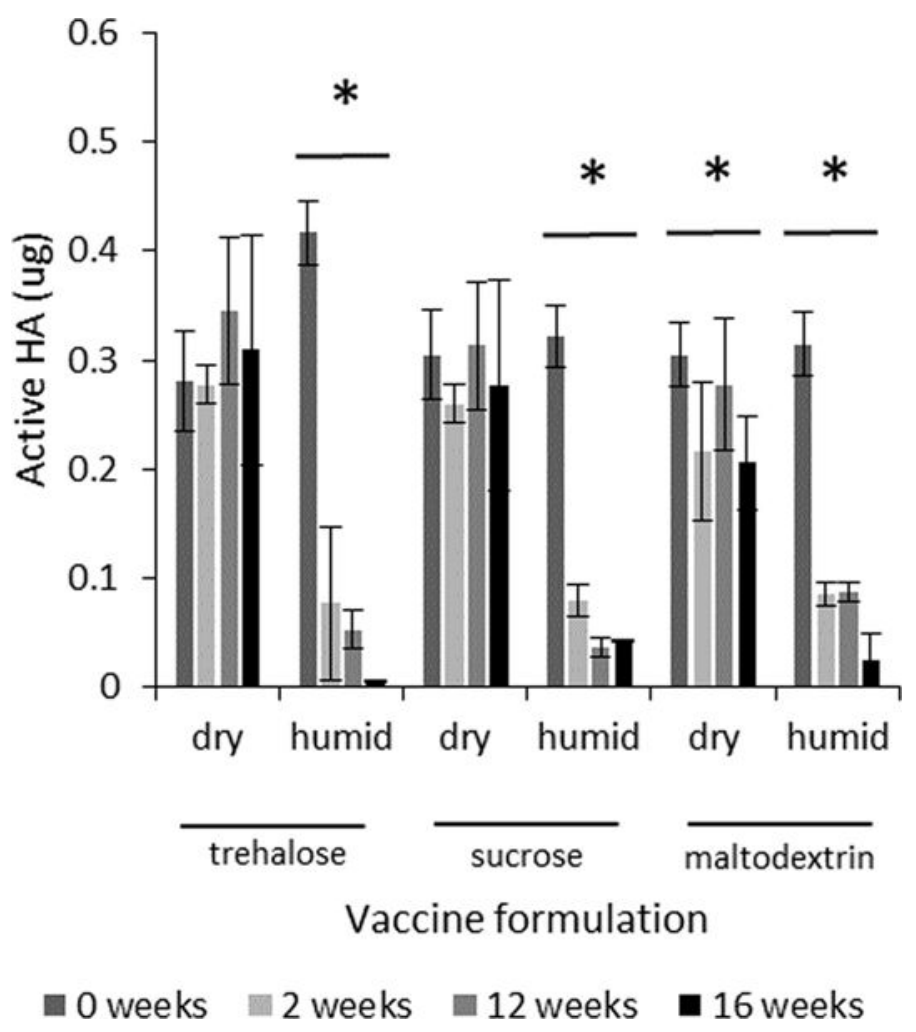

FIGURE 7.

Effect of humidity on HA activity after drying influenza vaccine on PDMS chips and storing with desiccant for up to 16 weeks at $40^{\circ} \mathrm{C}$. Vaccine (A/Brisbane/59/2007) was formulated with trehalose, sucrose or maltodextrin at a concentration of $10 \% \mathrm{w} / \mathrm{v}$ in ammonium acetate buffer. Chips were stored with either desiccant (dry) or silica gel that had been conditioned to maintain $70 \%$ relative humidity (humid). Asterisk (*) indicates a significant change in HA activity over time (ANOVA, $\mathrm{p}<0.05$ ). HA activity is shown as the mass of active HA. Data represent averages of $n=9$ replicates, with standard deviation bars shown. 


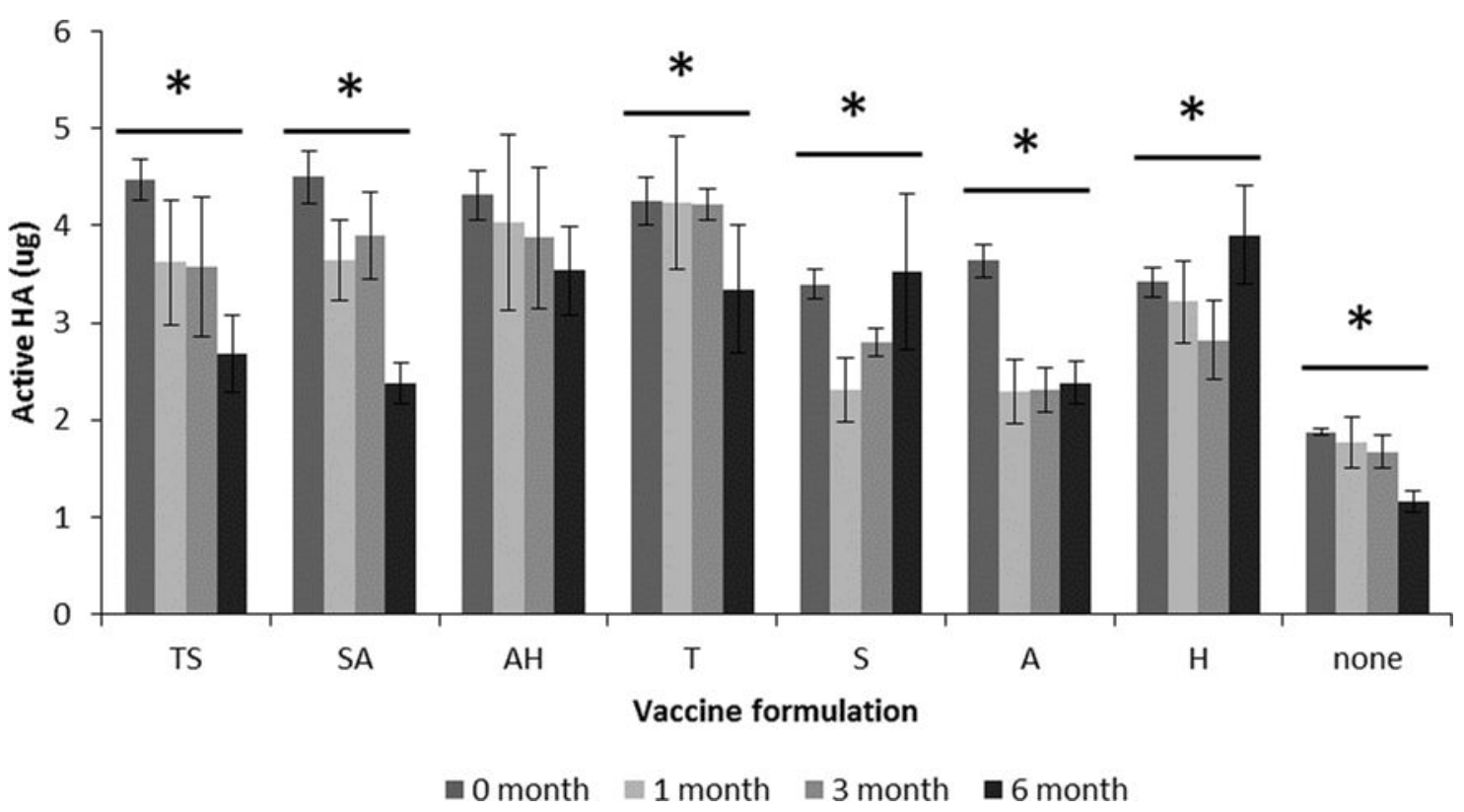

FIGURE 8.

Effect of formulation on HA activity after manufacturing microneedle patches and storing with desiccant for up to six months at $25^{\circ} \mathrm{C}$. Monovalent microneedle patches were produced with $\mathrm{B} /$ Brisbane/60/2008 influenza vaccine formulated with stabilizing excipients at a total concentration of $10 \% \mathrm{w} / \mathrm{v}$ in ammonium acetate buffer. The two-stabilizer combinations contained equal amounts of each excipient. $\mathrm{T}=$ trehalose, $\mathrm{S}=$ sucrose, $\mathrm{A}=$ arginine, $\mathrm{H}=$ sodium heptagluconate. $\mathrm{HA}$ activity is shown as the mass of active $\mathrm{HA}$ in each patch. Asterisk (*) indicates a significant change in HA activity over time (ANOVA, p < 0.05). Data represent averages of $n=6$ replicates, with standard deviation bars shown. 


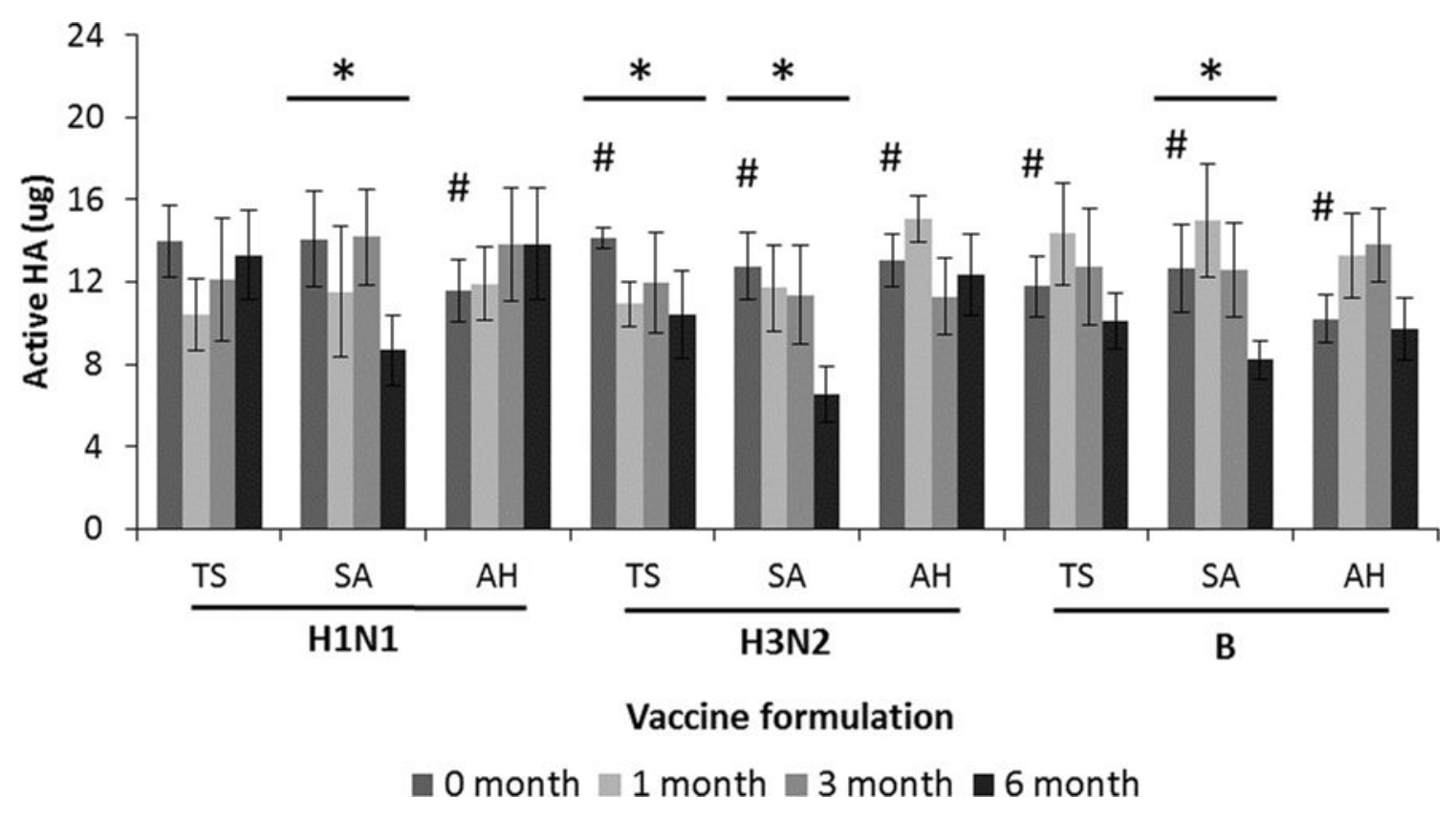

FIGURE 9.

Effect of formulation on Ha activity after manufacturing microneedle patches and storage with desiccant for up to six months at $25^{\circ} \mathrm{C}$. Trivalent microneedle patches were produced with A/Brisbane/59/2007 (H1N1), A/Victoria/210/2009 (H3N2) and B/Brisbane/60/2008 (B) influenza vaccine formulated with stabilizing excipients at a ratio of 50:50 with a total stabilizer concentration of $10 \% \mathrm{w} / \mathrm{v}$ in ammonium acetate buffer. $\mathrm{T}=$ trehalose, $\mathrm{S}=$ sucrose, $\mathrm{A}=$ arginine, $\mathrm{H}=$ sodium heptagluconate. $\mathrm{HA}$ activity is shown as the mass of active HA in each patch. Asterisk (*) indicates a significant change in HA activity over time (ANOVA, $p$ $<0.05$ ). Hash (\#) indicates the initial loading was significantly lower than the target dose of $15 \mu \mathrm{g}$ (Student's t-test, $\mathrm{p}<0.05$ ). Data represent averages of $\mathrm{n}=6$ replicates, with standard deviation bars shown. 